\author{
Victor Yavorsky and Zenoviy Znak
}

\title{
HYDROGEN SULFIDE DECOMPOSITION IN ULTRAHIGH-FREQUENCY PLASMA
}

\author{
Lviv Polytechnic National University \\ 12 S. Bandery str., 79013 Lviv, Ukraine
}

Received: J uly 08, 2009

(c) Yavorsky V., Znak Z., 2009

\begin{abstract}
The effect of gas rate on the plasma discharge stability and energy values of hydrogen sulfide decomposition has been established in plasma chemical reactor. Processes taking place in a swirling flow of hydrogen sulfide during its plasmolysis have been analyzed. Plasmotrone basic hydrodynamic model has been suggested.
\end{abstract}

Keywords: polymeric sulfur, hydrogen sulfide, plasma, plasmolysis.

\section{Introduction}

Polymeric sulfur belongs to the special kinds of sulfur possessing such specific properties as high reactivity, for instance $[1,2]$. Such property allows to use it as an effective vulcanizer for the production of industrial rubber products, automobile and aviation tires specifically [3]. Polymeric sulfur, compared with orthorhombic sulfur which is widely used now for tires production, improves physico-mechanical properties of the obtained products, e.g., rubber elasticity increases twice. This fact ensures better cohesion between the tires and road or landing strip; therefore the tires are more effective in all regimes, i.e. acceleration, braking, etc. The application of polymeric sulfur sharply improves integrity and, consequently, dependability of the tires, which are complex products consisting of up to 40 details. It allows to make vehicles safer and to reduce the accident rate. Operation time of the products on the basis of polymeric sulfur is actually twice higher than those based on orthorhombic sulfur. Pro tanto the bulks of used tires the utilization of which is still complicated in technological and ecological aspects, are reduced. The application of polymeric sulfur allows to use semi-automatic technological lines for tires production.

The sphere of polymeric sulfur application is amplified due to a sulfur chemical activity. It is used in construction industry [4], in electrochemical processes, for the production of radiation-resistant materials [5], and for the waste water treatment. Its application as an additive for concrete allows to increase considerably the hardening rate; therefore building products acquire strength only during some days whereas usually this process lasts more than a month. At the same time the products strength and hydrophilicity increase which allows to use polymeric sulfur for construction of civil and industrial objects as well as dams, collectors, etc. The application of polymeric sulfur as radiation-resistant material is very perspective because it may be used as individual substance or as a composite component with high values of ionizing radiation absorption factor.

The high consumption value of polymeric sulfur sets conditions for its high market value (more than $2500 \$ / \mathrm{t}$ ).

Nowadays polymeric sulfur is obtained mainly from melts. This method is complicated in technological aspect and imperfect in ecological one. Moreover, the main shortcoming is a low content of polymer in the product (less than 50-60\%). To obtain the product meeting the international standards the polymer content should be more than $90 \%$. Therefore the product must be purified from orthorhombic sulfur. Since the production of polymeric sulfur is imperfect, Ukraine and CIS countries import sulfur, mainly from Asian countries.

The main reason of existing technology imperfection is the fact that polymeric sulfur is obtained via polymerization of thermally activated orthorhombic sulfur [6], ring structures of which are destroyed with the formation of octa-atomic bi-radicals. Further they recombine with the formation of high-molecular product - polymeric sulfur. However, owing to the high viscosity of the medium the process is limited by diffusive phenomena. As a result, the main part of low-molecular fragments transfers again into a primary state orthorhombic modification (cyclic octasulfur).

The fundamentally new approach for the polymeric sulfur production is polymerization of atomic sulfur [6]. However the rational technology of atomic sulfur generation is necessary. Sulfur is atomized at temperatures above $2150 \mathrm{~K}$; the formed medium is very corrosive; therefore it is very difficult to implement the process 
commercially. We propose to obtain atomic sulfur via decomposition of sulfur-containing compounds. The hydrogen sulfur decomposition in ultrahigh-frequency plasma is one of such methods. We showed earlier $[7,8]$ that polymeric sulfur, the yield of which depends upon synthesis conditions, is formed as a result of the plasmolysis products cooling. Obviously the performance characteristics of the process will be also defined by energy costs for hydrogen sulfur decomposition.

So the aim of this work was to investigate hydrogen sulfide plasmolysis and to determine the energy performances of the process.

\section{Experimental}

Hydrogen sulfide plasmolysis was investigated using laboratory plasma chemical plant with nominal power of $2 \mathrm{~kW}$ [9], on the basis of standard magnetrone with radiation frequency of $2450 \pm 45 \mathrm{MHz}$. The power of ultrahigh-frequency radiation was varied from 0.8 to $1.8 \mathrm{~kW}$. The diameter of plasma chemical reactor made of quartz glass was $20 \mathrm{~mm}$. The plasma discharge was stabilized by a gasodynamic method. For this purpose hydrogen sulfide was tangentially introduced into plasmotrone. The value of plasma discharges was determined by a photographic method using colour filters. The temperature and pressure in different zones of plasma discharge were determined by a special sone.

The dissociation degree of hydrogen sulfide $(\alpha)$ was calculated by its concentration change which was determined by chromatography and volumetry before and after plasma chemical reactor.

$$
\alpha=\frac{C_{\text {out }}}{C_{0}}
$$

where $C_{0}$ and $C_{\text {out }}$ are hydrogen sulfide concentration at the reactor input and output, relatively, vol \%.

The energy performances of the process were determined by calorimetry. The corresponding scheme is represented in Fig. 1.

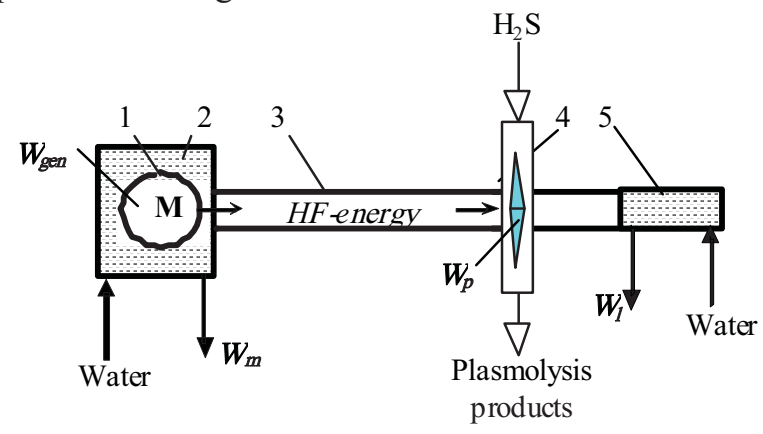

Fig. 1. The scheme of energy flows: magnetrone (1); magnetrone cooling system (2); ultrahigh-frequency (UHF) channel (3); reactor (4) and water load (5)
The power of radiation introduced into plasma discharge was calculated using the following formula:

$$
W_{p}=W_{g e n}-\left(W_{m}+W_{1}\right)
$$

where $W_{p}, W_{g e n}, W_{m}$ and $W_{1}$ are power introduced into plasma discharge, power of UHF generator, power eliminated from magnetrone by heat carrier (water) and power absorbed by water load under conditions of plasma discharge existence, respectively, $\mathrm{kW}$.

The power of UHF-generator was determined by a heat balance at the regime without plasma discharge:

$$
W_{g e n}=U I=W_{m}+W_{l}
$$

where $U$ - generator supply voltage, $\mathrm{V} ; I$ - current, A; $W_{m}^{\prime}$ - power eliminated from magnetrone by cooling water without plasma disharge, $\mathrm{kW} ; W_{1}^{\prime}$ - power absorbed by water load without plasma discharge, $\mathrm{kW}$.

The values of energy specific introduction into plasma discharge $(J, \mathrm{~kJ} / \mathrm{mol})$ and energy inputs for the production of $1 \mathrm{~mol}$ of the product $(A, \mathrm{~kJ} / \mathrm{mol})$ were calculated using Eqs. (4) and (5) [10]:

$$
\begin{gathered}
J=\frac{W_{p}}{V} \\
A=\frac{J}{\alpha}
\end{gathered}
$$

where $V$ - hydrogen sulfide consumption, $\mathrm{mol} / \mathrm{s} ; \alpha-$ decomposition degree of hydrogen sulfide.

\section{Results and Discussion}

The dependence of plasma discharge stability upon a gas flow rate has been established. The gas flow is fed to the reactor tangentially. At the reactor input, when the linear rate is less then $0.25 \mathrm{~m} / \mathrm{s}$, the discharge is unstable and without a robust contour. It localizes rapidly at the reactor wall mainly from the side where UHF radiation is supplied. It is well-known that plasma is characterized by electroconductivity. That is why it just absorbs UHFenergy supplied from the generator. The part of energy is consumed for molecular particles heating mainly due to the relaxation of excited particles. The result is the increase of temperature in the discharge zone. Since the discharge contacts with a reactor wall, the latter one is heated rapidly acquiring conductor properties and absorbing energy by itself. Then the reactor is overheated and destroyed.

In the range of such rates, even taking into consideration that at plasma temperature about $2000 \mathrm{~K}$ the gas flow rate increases sharply, it is impossible to implement the centrifugal effect in the reactor. Therefore conditions for plasma gasodynamic stability are absent in the plasmotrone. 
Within the temperature range of $0.25-0.32 \mathrm{~m} / \mathrm{s}$ the plasma stability is satisfactory but it is very sensitive to pressure fluctuations. Thus, a pressure pulsation may disturb the structure of plasma discharge and its placement in the reactor resulting in its displacement to the reaction wall. Therefore hydrogen sulfide supply rate must be stable in order to prevent plasma delocalization.

The increase of linear rate above $0.32 \mathrm{~m} / \mathrm{s}$ provides a high stability of plasma discharge in the reactor. It has a clear contour, narrows to $12-14 \mathrm{~mm}$ and lengthenes along a reactor vertical axis till $130-160 \mathrm{~mm}$. Slight pressure fluctuations or changes in gas rate virtually do not affect the pasmolysis proceeding.

The high stability of plasma discharge is caused by a considerable pressure gradient in the radial profile of plasmotrone due to the tangential supply of gas to the reactor. The zone of decreased pressure $(\Delta P=-740 \mathrm{~Pa})$ occurs along the reactor axis and the zone of increase pressure $(\Delta P=620 \mathrm{~Pa})$ - at periphery. Quantitative change of pressure by reactor radius may be expressed by the dependence (6), which is basic equation of hydrodynamic model of UHF-plasmotrone.

$$
P=P_{0}\left(1-(K-1) \rho_{0} I_{0}\left(8 K P_{0} \pi^{2} r^{2}\right)^{-1}\right)^{-2}
$$

where $P, P_{0}$ - pressure by reactor radius $r(\mathrm{~m})$ and initial pressure, Pa; $K$ - movement amount of flow; $\rho_{0}-$ initial density of medium, $\mathrm{kg} / \mathrm{m}^{3} ; I$ - intensity of a swirling flow.

$$
\begin{gathered}
K=2 \pi \int_{0}^{r} r\left(\rho \cdot u_{z}^{2}+P\right) d r \\
I_{0}=\frac{u_{y}}{2 \pi r}
\end{gathered}
$$

where $u_{y}$ and $u_{z}$ are radial and axial components of the swirling flow in plasmotrone, respectively.

Obviously, that just in the axial reactor zone (r!0) there are most favourable conditions for the plasma discharge initiation because just here the following condition takes place:

$$
E_{H F} / V \cdot N \geq E_{E X}
$$

where $E_{H F}$ - energy introduced into the reactor volume $V, \mathrm{~N}$ - number of particles in the reactor volume $V, E_{E X}{ }^{-}$ excitation energy of the particle.

Apparently, the plasma initiation is impossible at the reactor periphery, that gives the possibility to localize plasma inside the central area of the plasma chemical reactor.

Hydrogen sulfide decomposition is described by the sum equation (10):

$$
\mathrm{H}_{2} \mathrm{~S} \Leftrightarrow \mathrm{H}_{2}+\mathrm{S}
$$

However, in the plasma chemical system a great amount of intermediate products containing sulfide sulfur is formed. Hydrogen-sulfide radicals are primary products of hydrogen sulfide decomposition:

$$
\mathrm{H}_{2} \mathrm{~S} \Leftrightarrow \mathrm{HS}^{\bullet}+\mathrm{H}^{\bullet}
$$

Ion-molecules and ion-radicals of hydrogen sulfide are formed as a result of electron "adhesion". Electrons concentration in the plasma is very high.

$$
\begin{gathered}
\mathrm{H}_{2} \mathrm{~S}+\mathrm{e}^{-} \Leftrightarrow \mathrm{H}_{2} \mathrm{~S}^{-} \\
\mathrm{HS}^{-}+\mathrm{e}^{-} \Leftrightarrow \mathrm{HS}^{-}
\end{gathered}
$$

Positively charged ion-molecules and ion-radicals are also formed in the plasma discharge owing to the proceeding of ionization process:

$$
\begin{aligned}
& \mathrm{H}_{2} \mathrm{~S}-\mathrm{e}^{-} \Leftrightarrow \mathrm{H}_{2} \mathrm{~S}^{+} \\
& \mathrm{HS}^{\bullet}-\mathrm{e}^{-} \Leftrightarrow \mathrm{HS}^{\bullet+}
\end{aligned}
$$

The decomposition degree of hydrogen sulfide naturally increases with the increase of the value of energy introduced into the reaction system (Fig. 2).

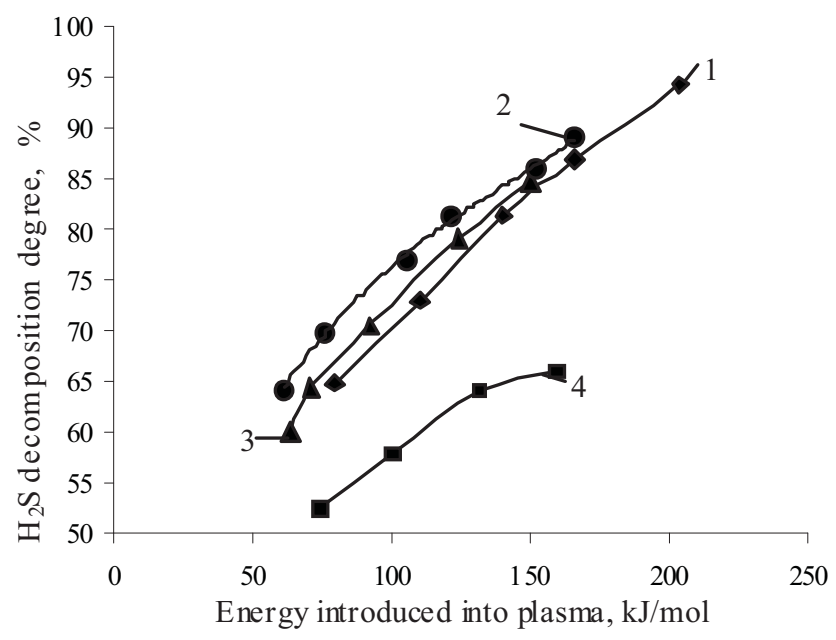

Fig. 2. Dependence of a decomposition degree of hydrogen sulfide upon the value of energy introducing into plasma at different gas linear rates in the reactor (normal conditions): 0.29 (1); 0.39 (2); 0.46 (3) and $0.56(4) \mathrm{m} / \mathrm{s}$

Within the range of gas velocities from 0.29 to 0.46 there is practically a linear dependence of hydrogen sulfide decomposition degree upon energy introduction value. Thus in the range of energy introduction of $55-170 \mathrm{~kJ} / \mathrm{mol}$ the decomposition degree increase is $22-30 \%$ and the total conversion is $91 \%$. Such linear dependence indicates that the energy of ultrahigh-frequency radiation is consumed for the proceeding of one-type processes as a rule. Taking into consideration the fact that during the interaction of UHF-radiation with a compound it transforms by three channels: chemical, excitation and oscillatory, we may assert that the first channel is the main one.

The diameter of the plasma filament within the mentioned rate range slightly decreases: in the area of discharge maximum diameter - by $1-3 \mathrm{~mm}$. The decrease of effective area of energy absorption should decrease 
the decomposition degree of hydrogen sulfide. Hence, the increase of decomposition degree of hydrogen sulfide may be most probably explained by the origination of back radial flows in the reactor. They originate due to the increase of a swirling degree of gas flow under the action of centrifugal forces. At the same time concentrations of heavier particles (atomic sulfur, low-molecular products of their recombination, nondissociated hydrogen sulfide, etc.) increase at the reactor periphery. It is the reason for the formation of a zone with increased pressure, so a radial gradient of pressures appears in the reactor. Since the rector temperature in this zone is lower (to $800 \mathrm{~K}$ ) than that in the central part (more than $2000 \mathrm{~K}$ ), the growth of low-molecular gaseous fragments of sulfur continues. Apparently the effect of centrifugal forces on these particles increases. Thus, in the plasmotrone such conditions occur under which a back flow (from the periphery to the centre) is formed due to the pressure gradient. The gradient in its turn is formed by lowmolecular sulfide particles, namely by nondissociated hydrogen sulfide and products of its partial dissociation (ion-molecules, radicals, ion-radicals, etc.). Therefore hydrogen sulfide in different forms returns to the plasma discharge, where its dissociation takes place. Here the decomposition degree increases.

At the same time one more important process takes place. Since the recombination processes are exothermic, some part of heat is transferred into a plasma discharge by sulfide particles. This heat also increases the decomposition degree of hydrogen sulfide - so called internal (i.e. inside the plasma chemical system) heat recovery takes place [11].

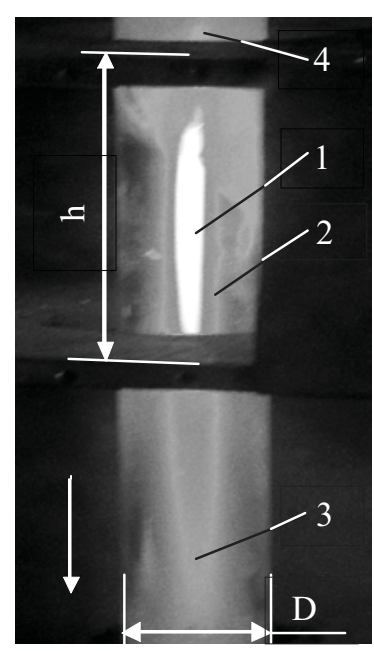

Fig. 3. Plasma discharge form in the reactor (photo is taken using a red filter). Areas of plasma discharge: central (1); external (2); outflow (low) (3) and upper (4). $D$ - reactor diameter; $h$ - channel height of UHF-energy introduction (arrow shows the direction of a gas movement)
At higher linear rates of hydrogen sulfide $(0.56 \mathrm{~m} / \mathrm{s})$ the plasma discharge concentrates along the reactor axis, its diameter does not exceed $8-12 \mathrm{~mm}$ and its length increases to $250-300 \mathrm{~mm}$ (Fig. 3). However the decomposition degree in the mentioned range decreases considerably and does not exceed $65 \%$.

Due to the red light filter we can legibly see the areas of plasma discharge: central (1) with the highest temperature and external sphere (2) with a lower temperature (1100$1300 \mathrm{~K})$. The central zone of the charge locates in the area of energy supply and does not exceed the height of UHFchannel $h$. The temperature of discharge outflow zone (3) ranges from 900 to $1200 \mathrm{~K}$. At gas rates above $0.4 \mathrm{~m} / \mathrm{s}$ the discharge lengthens and appears before UHF-channel. It points to the complex system of flows in the reactor.

At the gas rate of $0.56 \mathrm{~m} / \mathrm{s}$ the effective longitudinal section of plasma decreases but the degree of energy absorption by plasma determined by calorimetry actually does not change and equals to $82-84 \%$. Therefore the decrease of process performances can not be explained by the decrease of energy amount introduced into the plasma chemical system. Such negative effect may be explained by strengthening of centrifugal forces. First, it sets conditions for the intensive removal of nondissociated hydrogen sulfide from the plasma narrow zone. Second, the value of the back flow decreases, so the less amount of sulfide compounds returns for redecomposition. Third, the axial component of a swirling flow (Fig. 4) increases with the increase of gas linear rate and intensification of centrifugal phenomena. Pro tanto hydrogen sulfide decomposition products as well as sulfide compounds are faster removed from the reactor. At the same time the zones from plasma discharge remove a great amount of energy - effect of heat internal recovery attenuates sharply.

Such conclusion is confirmed by the dependence of energy consumption necessary for hydrogen sulfide decomposition upon the amount of introduced energy (Fig. 5).

Within a range of linear rates of $0.29-0.46 \mathrm{~m} / \mathrm{s}$ at equal values of introduced energy values of energy consumption differ slightly - by $3-5 \%$ (i.e. within a range of experimental uncertainty, which may be $7-10 \%$ for such processes). Thus, energy introduced into plasma discharge in this range of linear rates is most efficiently consumed for hydrogen sulfide decomposition: the increase of energy consumption is $0.75-0.80(\mathrm{~kJ} / \mathrm{mol} / \mathrm{kJ} /$ $\mathrm{mol}$ ). It means that $100 \mathrm{~kJ} / \mathrm{mol}$ are consumed for hydrogen sulfide decomposition at the introduction of energy in the amount of 75-80 kJ/mol. Such prima facie contradiction is explained by realization of the effect of heat internal recovery.

At a gas rate of $0.56 \mathrm{~m} / \mathrm{s}$ the increase of energy consumption rises to $1(\mathrm{~kJ} / \mathrm{mol} / \mathrm{kJ} / \mathrm{mol})$. That is why in such a case there is a greater necessity of energy for hydrogen sulfide decomposition (by $20 \%$ at the average). Thus energy is consumed less effectively. 


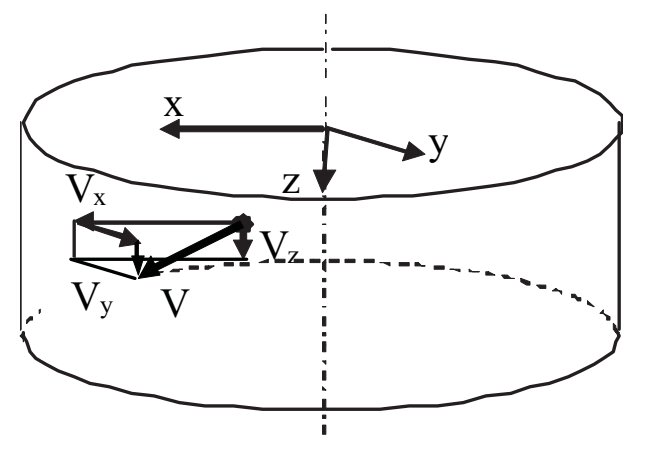

Fig. 4. Scheme of rates distribution in a swirling flow in the plasma chemical reactor: $\mathrm{V}_{\mathrm{x}}-$ radial; $\mathrm{V}_{\mathrm{y}}-$ tangential; $\mathrm{V}_{\mathrm{z}}-$ axial; $\mathrm{V}$ - resulting

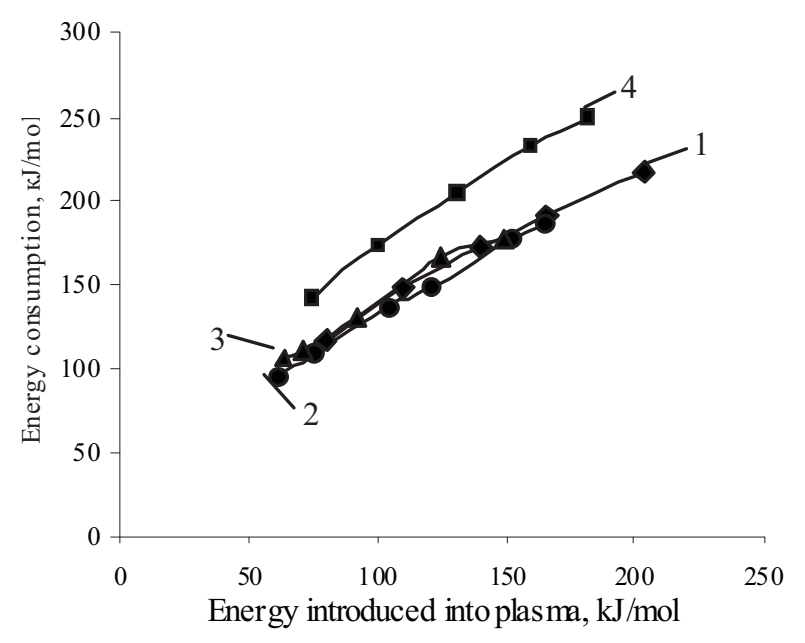

Fig. 5. Dependence of energy inputs upon energy values introduced into plasma at linear gas rate in the reactor (normal conditions): $0.29(1) ; 0.39(2) ; 0.46$ (3) and $0.56(4) \mathrm{m} / \mathrm{s}$

It would be logically predicted that polymer sulfur yield essentially depends upon decomposition degree of hydrogen sulfide, because the increase of the mentioned value increases concentration of atomic sulfur. Then it is obvious that under corresponding conditions the recombination rate of sulfur atoms (which are bi-radicals by their nature) is proportional to their concentrations. Thus, in order to achieve the considerable yield of polymeric sulfur it is necessary to ensure the maximum possible conversion of hydrogen sulfide. For this aim it is necessary to increase the value of energy introduced into the plasma chemical system. The increase of introduced energy is achieved by the increase of UHF-generator capacity followed with the increase energy consumption for hydrogen sulfide plasmolysis. Obviously, this fact affects the technico-economic performances of hydrogen sulfide decomposition with the production of polymeric sulfur. Therefore, the dependence of specific energy consumption necessary for hydrogen sulfide plasmolysis upon the decomposition degree has been determined (Fig. 6). Obtained results confirm the earlier stated conclusion about the efficiency decrease of UHF-energy by plasma discharge with the increase of gas flow linear rate in the reactor. Within the range of $0.29-0.46 \mathrm{~m} / \mathrm{s}$ rates, in order to achieve the same decomposition degree of hydrogen sulfide, specific energy consumption is also the same. Thus, for example, decomposition degree of $70 \%$ is achieved in the range of specific energy consumption 1.3$1.6 \mathrm{~kW} / \mathrm{h} \cdot \mathrm{m}^{3}$ and $85 \%$ - in a narrower range of 2.1 $2.25 \mathrm{~kW} / \mathrm{h} \cdot \mathrm{m}^{3}$.

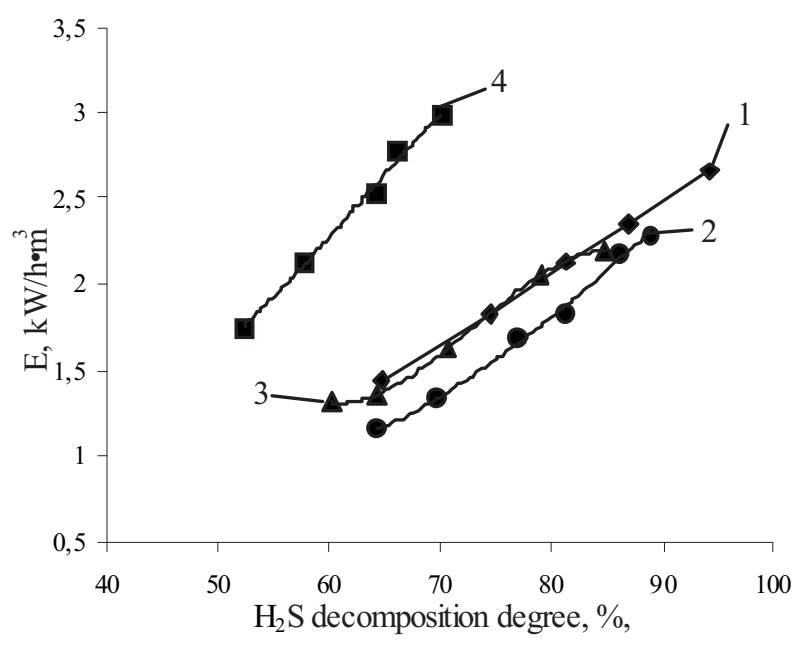

Fig. 6. Dependence of specific energy consumption $\left(\mathrm{E}, \mathrm{kW} / \mathrm{h} \cdot \mathrm{m}^{3}\right)$ upon decomposition degree of hydrogen sulfide at the gas linear rate (normal conditions): 0.29 (1); $0.39(2) ; 0.46(3)$ and $0.56(4) \mathrm{m} / \mathrm{s}$

The analysis of results from Fig. 6 allows to assert that at gas linear rate more than $0.5 \mathrm{~m} / \mathrm{s}$ the specific energy consumption increases sharply. At hydrogen sulfide rate of $0.56 \mathrm{~m} / \mathrm{s}$ the decomposition degree $(70 \%)$ needs approximately $3 \mathrm{~kW} / \mathrm{h} \cdot \mathrm{m}^{3}$. Actually it is twice more than that within the rate range of $0.29-0.46 \mathrm{~m} / \mathrm{s}$. At the same time, if we compare results for 0.46 and $0.56 \mathrm{~m} / \mathrm{s}$ we can see that plasmotrone productivity increases only by 17 $18 \%$ with the increase of the mentioned rates.

On the basis of experimental results the optimum range of hydrogen sulfide rates $(0.34-0.46 \mathrm{~m} / \mathrm{s})$ at the input of plasma chemical reactor has been determined. Within the mentioned range the plasma discharge is characterized by high stability. Specific energy consumption is approximately $2 \mathrm{~kW} / \mathrm{h} \cdot \mathrm{m}^{3}$ in order to achieve decomposition degree of hydrogen sulfide of $85-90 \%$.

\section{Conclusions}

The stability of plasma discharge in UHFplasmotrones is achieved by the creation of the swirling 
gas flow at its linear rate above $0.25 \mathrm{~m} / \mathrm{s}$. The increase of decomposition degree of hydrogen sulfide with the increase of gas rate from 0.29 to $0.46 \mathrm{~m} / \mathrm{s}$ is explained by the effect of heat internal recovery which is defined by the presence of recirculation flows in the reactor.

\section{References}

[1] Menkovsky M. and Yavorsky V. Technolodiya sery. Khimiya, Moskwa 1985.

[2] Meyer B.: Sulfur, energy and environment. AmsterdamOxford-New York 1977.

[3] Kuznetsov A., Kulikova O. and Poddubnui I. Intern. Polym. Sci. and Techn. 2002, 29, 1.

[4] Fomin A., Porfir' eva R. and Kchozin V. Vestn. Kazan. Univ., 2001, 2, 191.

[5] Orlovsky Y.I., Proshin A.I. Izvestiya vyzov. Stroitel'stvo. 2004, 9. 21.

[6] Yavorsky V. Chemistry \& chemical technology. 2008. 2, 2. 129.

[7] Znak Z.O. Ekotechnologii I Resursosberezhenie, 2006, 5, 44.
[8] Znak Z.O., Yavorsky V.T., Olenych R.R. Voprosy Khimii i Khim. Techn., 2005, 3, 66.

[9] Znak Z.O., Yavorsky V.T. Vostochnoevropeisky Zyrn. Peredovuch Technologiy. 2006, 4/3 (22), 76.

[10] Nester S.A., Potapkin B.V., Levitsky A.A. etc. Kinetikostatiaticheskoe modelirovanie kchimicheskich reaktsiy $\mathrm{v}$ gazovom razr'ade. CNIIatominform, Moskva. 1988.

[11] Macheret S.O., Rysanov V.D., Fridman A.A. Zurn. Techn. Fiziki . 1987. 57. 4. 712.

\section{ДОСЛЦЖЕННЯ ПРОЦЕСУ РОЗКЛАДУ СІРКОВОДНЮ В НАДВИСОКОЧАСТОТНІЙ ПЛАЗМІ}

Анотація. Встановлено вплив швидкості газу в плазмохімічному реакторі на стабільність плазмового розряду та енергетичні показники процесу розкладу сірководню. Проаналізовано процеси, щуо відбуваються в закрученому потоия сірководню під час його плазмолізу. Запропоновано базову гідродинамічну модель плазмотрона.

Ключові слова: полімерна сірка, сірководень, плазма, плазмоліз 